\title{
SÍNTESE, CARACTERIZAÇÃO E ESTUDO REOLÓGICO DE MELHORADORES DO ÍNDICE DE VISCOSIDADE PARA ÓLEOS LUBRIFICANTES AUTOMOTIVOS
}

\author{
Maria Elisa Scarpelli Ribeiro e Silva ${ }^{1}$, Alessandra Chagas Daniel ${ }^{1}$, Roberto Fernando de \\ Souza Freitas ${ }^{1}$ e Ricardo Geraldo de Sousa ${ }^{1}$
}

${ }^{1}$ Laboratório de Ciência e Tecnologia de Polímeros, Departamento de Engenharia Química, Universidade Federal de Minas Gerais

E-mails: elisa@deq.ufmg.br, alessandrachagasdaniel@gmail.com, freitas@ deq.ufmg.br, rick@deq.ufmg.br

\section{RESUMO}

Melhoradores do índice de viscosidade (MIVs) são essenciais para o bom desempenho dos óleos lubrificantes automotivos devido à sua capacidade de reduzir a variação da viscosidade com a temperatura. Neste trabalho foram sintetizados terpolímeros melhoradores do índice de viscosidade em óleo mineral básico, a partir dos monômeros dodecilmetacrilato, octadecilmetacrilato e anidrido maleico, utilizando-se como iniciador o Luperox ${ }^{\circledR}$ TBEC, em temperaturas de $100^{\circ} \mathrm{C}$. Seis sistemas, com concentrações que variaram de 2 a $4 \%$ de anidrido maleico, de 42 a $45 \%$ de dodecilmetacrilato e de 53 a $56 \%$ de octadecilmetacrilato, foram sintetizados e caracterizados por FTIR e TG. O comportamento reológico das amostras foi estudado a partir das viscosidades cinemáticas, índices de viscosidade (IVs), estabilidade ao cisalhamento e testes de fluxo em estado estacionário. A ausência de bandas típicas de insaturação de carbono dos monômeros nos espectros de FTIR indicou a formação dos MIVs. Os resultados da TG mostraram que as amostras aditivadas apresentaram maior estabilidade térmica do que o óleo puro. A estabilidade térmica dos compostos foi ligeiramente afetada pela variação das concentrações de anidrido maleico e octadecilmetacrilato. Entretanto, mudanças nas concentrações de dodecilmetacrilato promoveram alterações mais significativas. Os valores de viscosidade cinemática aumentaram em comparação com o óleo base, em virtude da capacidade de espessamento dos MIVs. Todas as amostras aditivadas apresentaram um aumento em seus IVs relativamente ao do óleo puro, sendo este aumento significativamente dependente da concentração do anidrido maleico. Os IVs mantiveram-se elevados mesmo após os testes de cisalhamento. O comportamento do fluido foi bem ajustado pelo Modelo da Lei de Potência. 


\section{INTRODUÇÃO}

A demanda por inovações tecnológicas na área de óleos lubrificantes tem sido crescente em função da evolução dos motores automotivos. Atualmente, várias propriedades dos lubrificantes têm sido reforçadas ou criadas por meio de aditivos químicos poliméricos, em busca constante de aumento de desempenho, rendimento, durabilidade e na redução das emissões e dos custos de operação dos motores automotivos [1].

Nos últimos anos, o Brasil tem se destacado no setor tanto por possuir tecnologia de ponta para exploração e refino de petróleo, quanto por ser um mercado promissor para venda de óleos lubrificantes. De acordo com BARROS [2], o país consumiu 3,2\% de todo o volume do produto comercializado no mundo no ano de 2009 e espera-se que a demanda nacional registre crescimentos expressivos até 2019. É possível prever, neste cenário, oportunidade de desenvolvimento de know-how em tecnologia de aditivos químicos para óleos lubrificantes automotivos para atendimento ao mercado.

Os melhoradores de índice de viscosidade (MIVs) se encontram entre os principais tipos de aditivos utilizados em formulações de óleos lubrificantes automotivos [3]. São aditivos poliméricos que têm por objetivo minimizar a variação da viscosidade do óleo com a variação da temperatura. Essa função é de grande importância, pois os motores automotivos são submetidos a altas temperaturas, o que pode comprometer a lubrificação do equipamento.

Dentre os MIVs comerciais geralmente usados em lubrificantes automotivos, segundo MORTIER et al. [4], destacam-se os polimetacrilatos de alquila, os poli(etileno-copropileno), os poli(estireno-co-butadieno ou isopreno) hidrogenados e suas modificações e uma combinação dos dois primeiros.

O índice de viscosidade (IV) foi primeiramente proposto por Dean e Davis em 1929 para representar o efeito da temperatura sobre a viscosidade cinemática de óleos básicos e lubrificantes [4][5]. Desde essa proposição, pesquisadores e profissionais da área de lubrificantes e de refinarias de petróleo quantificam a influência da temperatura sobre a viscosidade utilizando o IV como principal parâmetro de determinação da qualidade de lubrificação e definem as possíveis aplicações de um óleo em uma ampla faixa de temperatura [6]. Quanto maior for o IV do fluido, menos a viscosidade variará para uma dada mudança de temperatura [7].

Segundo relatado por OLIVEIRA [6], "face à importância técnica, econômica e ambiental dos aditivos melhoradores do índice de viscosidade, vários trabalhos vêm sendo desenvolvidos, alguns na área acadêmica e muitos deles nos centros de pesquisa e desenvolvimento de empresas diversas". O desenvolvimento de uma formulação adequada deve considerar dois aspectos básicos relacionados ao desempenho dos MIVs: as suas propriedades como melhoradores do índice de viscosidade e o seu comportamento quando submetidos a elevadas taxas de cisalhamento.

Neste trabalho foram sintetizados seis MIVs baseados em terpolímeros de dodecilmetacrilato, octadecilmetacrilato e, como terceiro comonômero, o anidrido maleico, tendo como solvente, óleo mineral básico. Os sistemas foram caracterizados utilizando-se as técnicas de espectroscopia de absorção molecular na região do infravermelho por transformada de Fourier e termogravimetria, e avaliados em relação aos seu desempenho por meio das determinações 
das viscosidades cinemáticas a 40 e $100^{\circ} \mathrm{C}$, do índice de viscosidade, da estabilidade ao cisalhamento e por medidas reológicas.

\section{MATERIAIS E MÉTODOS}

\subsection{Materiais}

Os MIVs foram sintetizados a partir dos monômeros dodecilmetacrilato (DC), octadecilmetacrilato (OC) e anidrido maleico (AM). Como iniciador foi utilizado o Luperox ${ }^{\circledR}$ TBEC (LP). As sínteses foram efetuadas em óleo mineral básico, na temperatura de $100^{\circ} \mathrm{C}$, por 5 horas. Na Tabela 1 estão sumariados os valores das concentrações utilizadas, em massa, dos monômeros e do iniciador.

Tabela 1 - Concentrações dos monômeros e do iniciador utilizadas nas sínteses dos MIVs.

\begin{tabular}{ccccc}
\hline & \multicolumn{2}{c}{ Concentração de Monômeros $\left(\% \mathrm{~m}^{-1}\right)$} & $\begin{array}{c}\text { Iniciador } \\
\left(\% \mathrm{~m}^{-1}\right)\end{array}$ \\
\cline { 2 - 5 } Amostra & AM & DC & OC & LP \\
\hline A.1 & 2 & 42 & 56 & 2 \\
\hline A.2 & 3 & 42 & 55 & 2 \\
\hline A.3 & 4 & 42 & 54 & 2 \\
\hline A.4 & 2 & 45 & 53 & 2 \\
\hline A.5 & 3 & 44 & 53 & 2 \\
\hline A.6 & 4 & 43 & 53 & 2 \\
\hline
\end{tabular}

\subsection{Métodos}

\subsubsection{Preparação das amostras}

Todas as 06 amostras dos MIVs sintetizados foram diluídas a 5\% $\left(\mathrm{m} \cdot \mathrm{m}^{-1}\right) \mathrm{em}$ óleo básico, em chapa aquecedora com agitação por, aproximadamente, 30 minutos a $40^{\circ} \mathrm{C}$.

\subsubsection{Caracterização dos óleos aditivados por FTIR e TG}

Os espectros de absorção molecular no infravermelho, na faixa de 4000 a $650 \mathrm{~cm}^{-1}$, foram obtidos em espectrômetro FTIR modelo Nicolet 6700 da Thermo Fisher Scientific, no modo ATR, com 64 varreduras e resolução de $4 \mathrm{~cm}^{-1}$.

Para as análises termogravimétricas utilizou-se o equipamento Shimadzu TGA-50, com a seguinte programação: aquecimento de temperatura ambiente $\left(20^{\circ} \mathrm{C}\right)$ até $500^{\circ} \mathrm{C}$, com razão de aquecimento de $10^{\circ} \mathrm{C} \cdot \mathrm{min}^{-1}$, 
utilizando nitrogênio como gás de arraste, com vazão de $50 \mathrm{~mL} \cdot \mathrm{min}^{-1}$, em porta amostra de platina. A massa da amostra variou em torno de 5,00 mg.

\subsubsection{Caracterização reológica dos óleos lubrificantes puro e aditivados}

As viscosidades cinemáticas das amostras foram obtidas a $40^{\circ} \mathrm{C}$ e $100^{\circ} \mathrm{C}$ de acordo com a norma ASTM D445, em viscosímetros Cannon Fenske calibrados, em banho de silicone.

Os índices de viscosidade foram calculados de acordo com a norma ASTM D2270, a partir dos resultados obtidos no teste de viscosidade cinemática.

Os testes reológicos de fluxo em regime permanente foram realizados em reômetro AR-G2 da TA Instruments. A tensão de cisalhamento foi variada entre 0 e $280 \mathrm{~Pa}$, na temperatura de $25^{\circ} \mathrm{C}$.

\section{RESULTADOS E DISCUSSÃO}

Na Figura 1 é mostrado um espectro de FTIR típico, representativo dos espectros de todas as amostras aditivadas.

Figura 1. Espectro de FTIR para a amostra A1

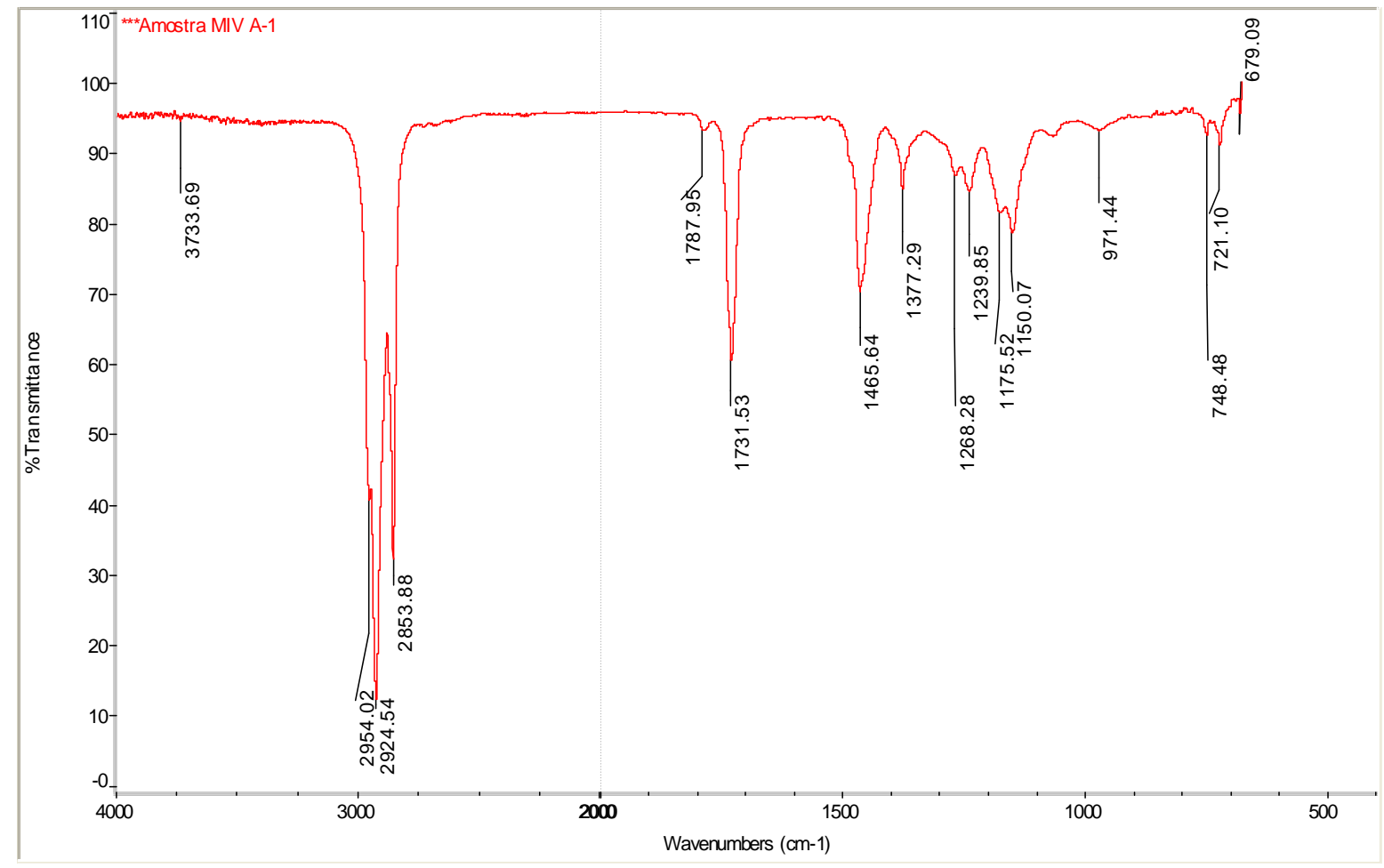

A síntese dos terpolímeros pôde ser confirmada pela ausência das insaturações $\mathrm{C}=\mathrm{C}$ do dodecilmetacrilato e do octadecilmetacrilato, em $1639 \mathrm{~cm}^{-1}$, e em $1634 \mathrm{~cm}^{-1}$ do anidrido maleico, presentes nos monômeros puros. A deformação axial $\mathrm{C}=\mathrm{O}$ do anidrido maleico em $1779 \mathrm{~cm}^{-1}$ foi deslocada devido à ausência da dupla ligação na molécula. As bandas 
identificadas são características de polimetacrilatos, com exceção da deformação em 1787 $\mathrm{cm}^{-1}$, que é característica do anidrido maleico.

A termogravimetria possibilitou analisar a estabilidade térmica dos óleos puro e aditivados por meio da determinação das temperaturas de início $\left(\mathrm{T}_{\mathrm{i}}\right)$ e de máxima degradação $\left(\mathrm{T}_{\mathrm{dmax}}\right)$. Na Figura 2 são mostradas as curvas TG do óleo puro e das amostras aditivadas e na Tabela 2 estão sumariadas os valores das $\mathrm{T}_{\mathrm{i}}, \mathrm{T}_{\max }$ e a $\%$ de perda da massa.

Figura 2. Curvas TG do óleo puro e das amostras aditivadas

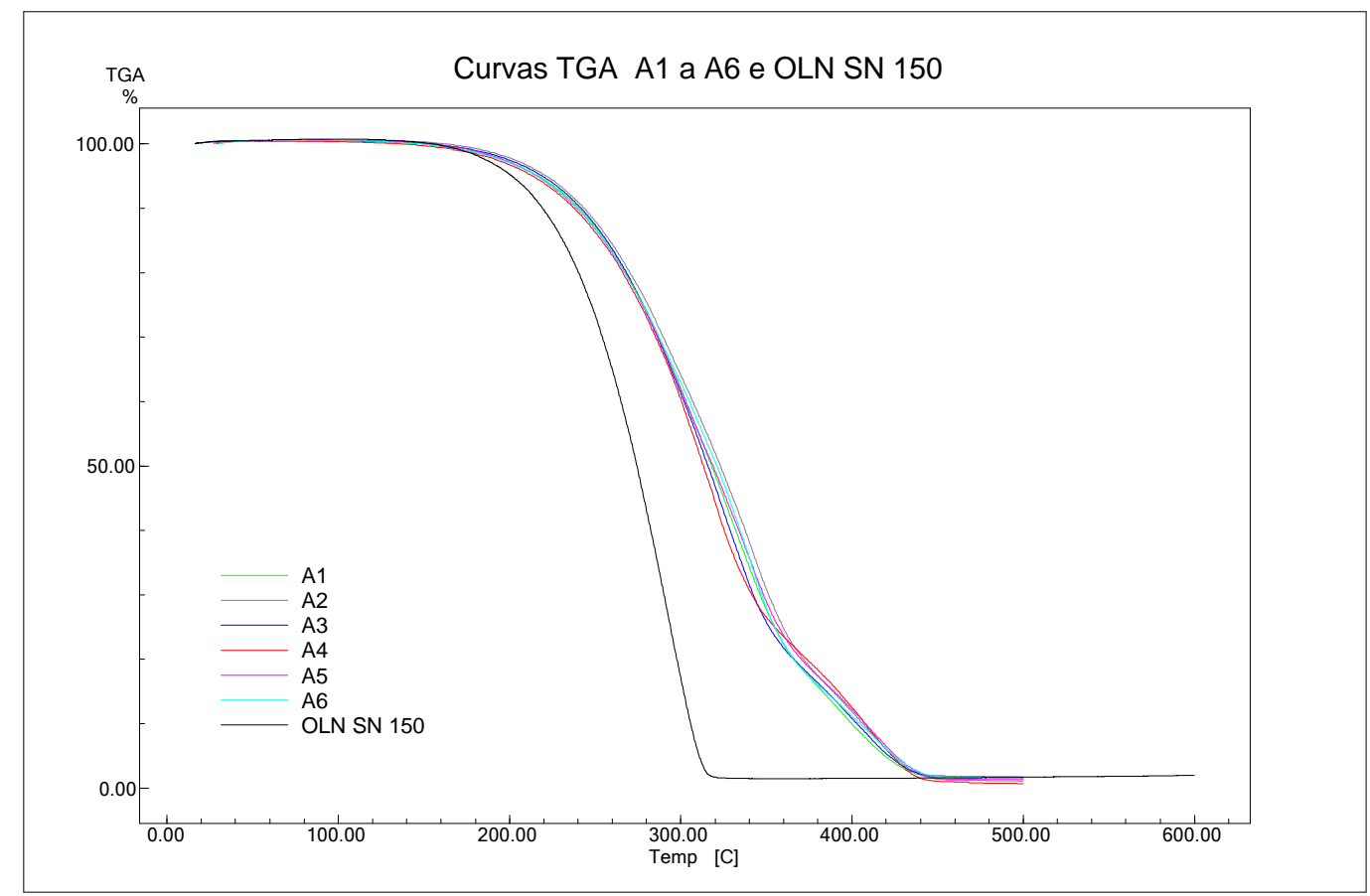

Observa-se que a degradação térmica do óleo puro se dá em apenas um estágio, enquanto nas amostras aditivadas percebe-se um segundo evento de perda de massa em temperaturas mais elevadas, provavelmente associado à degradação dos MIVs. 
Tabela 2. Resultados de TG do óleo puro e das amostras aditivadas

\begin{tabular}{cccc}
\hline Amostra & $\mathrm{T}_{\mathrm{i}}\left({ }^{\circ} \mathrm{C}\right)$ & $\mathrm{T}_{\max }\left({ }^{\circ} \mathrm{C}\right)$ & $\begin{array}{c}\text { Perda de } \\
\text { Massa }(\%)\end{array}$ \\
\hline Óleo SN-150 & 229 & 270 & 98 \\
\hline A1 & 256 & 328 & 99 \\
\hline A2 & 263 & 341 & 99 \\
\hline A3 & 256 & 318 & 98 \\
\hline A4 & 260 & 317 & 99 \\
\hline A5 & 238 & 290 & 99 \\
\hline A6 & 262 & 332 & 98
\end{tabular}

Todas as amostras aditivadas apresentaram temperaturas iniciais de degradação acima da obtida para o óleo puro $\left(229^{\circ} \mathrm{C}\right)$, variando de $238^{\circ} \mathrm{C}$ até $263^{\circ} \mathrm{C}$. Dessa maneira, é possível afirmar que a adição dos copolímeros estabilizou termicamente o óleo puro, possibilitando a utilização dos mesmos em motores automotivos, cuja temperatura de trabalho se situa em torno de $150^{\circ} \mathrm{C}$ [8]. São relatados, na literatura, diversos trabalhos que avaliam a relação entre o aumento da estabilidade térmica dos óleos lubrificantes automotivos e a estabilidade térmica dos polímeros [9] melhoradores do índice de viscosidade. Sabe-se que esta, dentre outros fatores, depende massa molar dos polímeros, da sua estrutura, da força de suas ligações químicas e do número de ramificações. Adicionalmente, no caso de copolímeros, deve-se destacar a importância da ordem de incorporação dos monômeros que, por sua vez, depende das reatividades relativas desses, levando a produtos com diferentes estruturas. No presente caso, verificou-se que a estabilidade térmica dos compostos foi influenciada pela concentração relativa dos monômeros, particularmente pela presença do dodecilmetacrilato.

Os resultados das medidas de viscosidade cinemática a $40^{\circ} \mathrm{C}$ e $100^{\circ} \mathrm{C}$ e dos IVs do óleo puro e das amostras aditivadas são mostrados na Tabela 3 . 
Tabela 3. Valores das viscosidades cinemáticas e dos índices de viscosidade do óleo puro e das amostras aditivadas

\begin{tabular}{cccc}
\hline Amostra & $\begin{array}{c}\text { Viscosidade } \\
\text { Cinemárica } \\
40{ }^{\circ} \mathrm{C}(\mathrm{cSt})\end{array}$ & $\begin{array}{c}\text { Viscosidade } \\
\text { Cinemática } \\
100{ }^{\circ} \mathrm{C}(\mathrm{cSt})\end{array}$ & $\begin{array}{c}\text { Índice de } \\
\text { Viscosidade }\end{array}$ \\
\hline Óleo puro & 31,0 & 5,2 & 102 \\
\hline A1 & 61,7 & 10,8 & 168 \\
\hline A2 & 48,6 & 8,4 & 150 \\
\hline A3 & 52,8 & 9,1 & 156 \\
\hline A4 & 58,0 & 10,1 & 164 \\
\hline A5 & 38,0 & 6,5 & 124 \\
\hline A6 & 50,2 & 8,8 & 156 \\
\hline
\end{tabular}

Para todos os sistemas aditivados houve aumento das viscosidades cinemáticas a 40 e a $100^{\circ} \mathrm{C}$ em relação ao óleo puro, evidenciando a capacidade de espessamento dos MIVs. Adicionalmente, verificou-se uma diminuição da viscosidade com o aumento da temperatura, comportamento típico de um sistema solúvel. Sabe-se que em um "bom" solvente, as cadeias poliméricas já se encontram em um estado "desenovelado". Um aumento da temperatura causa um enfraquecimento nas ligações polímero-solvente, possibilitando um aumento das interações intra e intermoleculares, resultando em um enovelamento das moléculas poliméricas, com a consequente redução da viscosidade. No caso de um solvente "pobre", o comportamento é oposto, isto é, a viscosidade aumenta com o aumento da temperatura. Nesse caso, com a elevação da temperatura, a força das interações intra e intermoleculares diminui, acarretando um aumento no volume hidrodinâmico das moléculas poliméricas e, também, da viscosidade. COVITCH e TRICKETT [10] descreveram o comportamento de vários polímeros nos chamados solventes "pobres", relatando as interações atrativas entre os segmentos de polímero. Contrariamente, na presença de "bons" solventes, segundo os autores, forças repulsivas entre segmentos de polímero são predominantes, resultando numa expansão das cadeias. Quando do aumento da temperatura, as forças repulsivas entre os polímeros e o solvente tornam-se mais eficazes, reduzindo a viscosidade.

.MORGAN et al. [11] relataram a diminuição da viscosidade com o aumento da temperatura em "bons" solventes, afirmando que a adição de pequenas quantidades de melhoradores do índice de viscosidade em óleos lubrificantes são capazes de reduzir eficazmente essa variação, em virtude do seu poder espessante.

Para todos os óleos aditivados foi observado um aumento nos IVs em relação ao óleo puro (IV=102), conforme esperado para um polímero melhorador do índice de viscosidade.

Os valores dos índices de viscosidades antes e após o teste de estabilidade ao cisalhamento são mostrados na Tabela 4. 
Tabela 4. Resultados dos IVs antes e após os teste de estabilidade ao cisalhamento do óleo puro e das amostras aditivadas

\begin{tabular}{ccc}
\hline MIV & IV antes & IV depois \\
\hline Óleo puro & 102 & 101 \\
\hline A1 & 168 & 146 \\
\hline A2 & 150 & 140 \\
\hline A3 & 156 & 143 \\
\hline A4 & 167 & 136 \\
\hline A5 & 126 & 122 \\
\hline A6 & 158 & 145 \\
\hline
\end{tabular}

As perdas permanentes de viscosidade (PVL) foram calculados conforme ASTM D 6278. Na Tabela 5 são mostrados os valores dos IVs e dos PVL (\%) dos óleos lubrificantes aditivados com os MIVs sintetizados.

Tabela 5. Índice de viscosidade (IV) e perda permanente de viscosidade (PVL) dos óleos lubrificantes aditivados com os MIVs sintetizados

\begin{tabular}{ccc}
\hline MIV & IV & PVL(\%) \\
\hline Óleo puro & 102 & --- \\
\hline A1 & 168 & 24,0 \\
\hline A2 & 150 & 11,1 \\
\hline A3 & 156 & 13,8 \\
\hline A4 & 167 & 21,3 \\
\hline A5 & 126 & 1,7 \\
\hline A6 & 158 & 12,6 \\
\hline
\end{tabular}

Observa-se que os MIVs apresentaram valores elevados para os IVs, mesmo após os testes de cisalhamento, valores estes superiores aos relatados por OLIVEIRA [6], para sistemas semelhantes.

As curvas relativas aos ensaios de fluxo em regime permanente (Steady State Flow) foram obtidas na temperatura de $25^{\circ} \mathrm{C}$, entre as tensões de cisalhamento de 0 a $280 \mathrm{~Pa}$. Na Figura 3 são mostradas as curvas de viscosidade dinâmica versus tensão de cisalhamento para as amostras sintetizadas. 
Figura 3. Curvas de viscosidade em função da tensão de cisalhamento em temperatura de $25^{\circ} \mathrm{C}$ para os óleos estudados

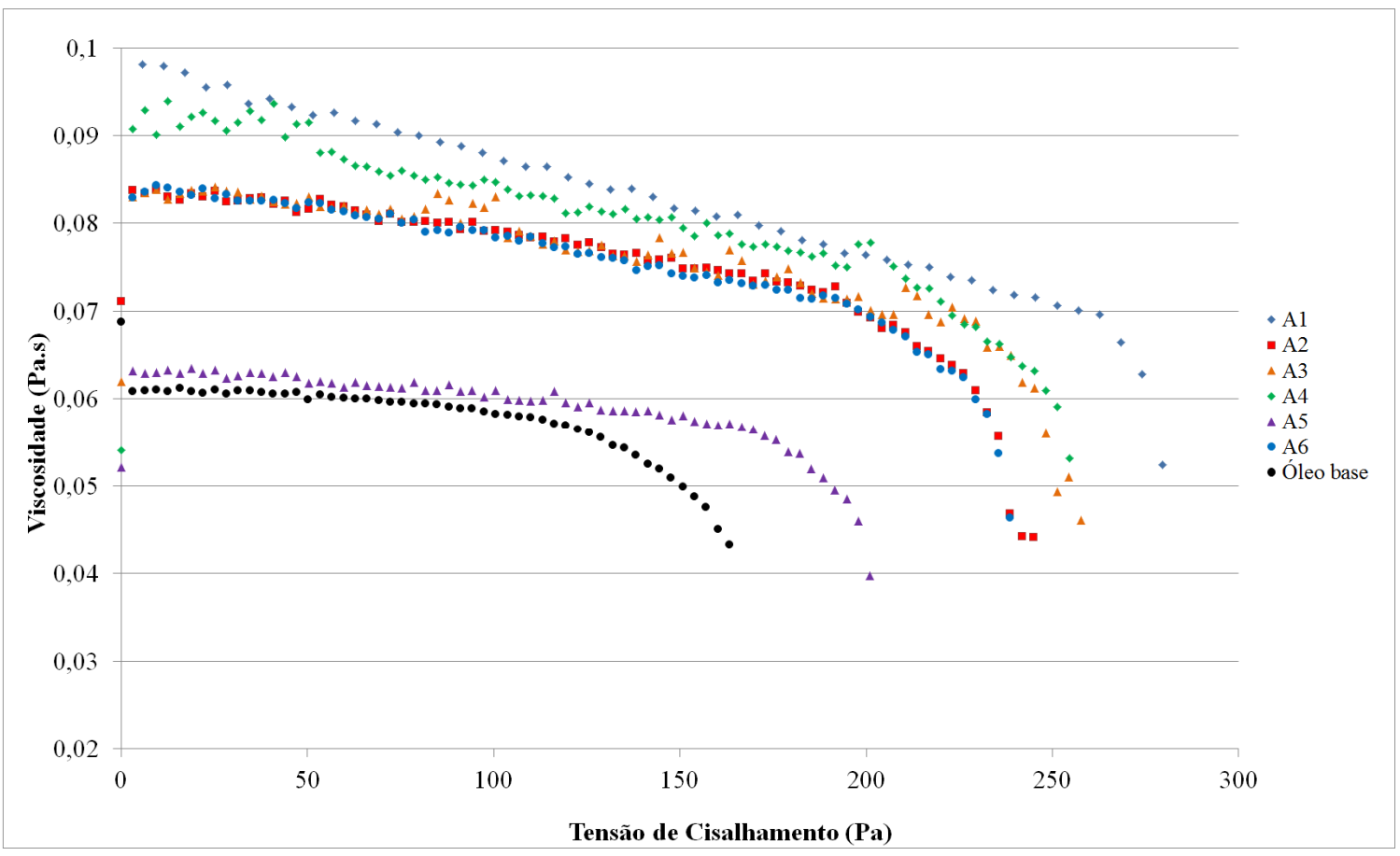

Observa-se que as viscosidades decrescem gradativamente com o aumento da tensão de cisalhamento. Percebe-se, também, que a baixas tensões de cisalhamento, as viscosidades permanecem constantes. Esse comportamento pode ser explicado considerando-se que em uma faixa de baixa tensão de cisalhamento, as moléculas poliméricas se mantêm dispersas aleatoriamente, devido ao movimento Browniano, apesar dos efeitos iniciais da orientação de cisalhamento. Em tensões de cisalhamento $(\tau)$ muito baixas, os fluidos pseudoplásticos se comportam similarmente aos fluidos Newtonianos, tendo uma viscosidade $\eta_{0}$ independente da tensão de cisalhamento. Quando $\tau$ aumenta a um ponto em que o cisalhamento, que induz a orientação da molécula polimérica, excede o efeito aleatório do movimento Browniano, a viscosidade diminui. Dessa forma, concluiu-se que as amostras estudadas podem apresentar tanto comportamento Newtoniano - a baixas tensões de cisalhamento, como não Newtoniano em valores de $\tau$ mais altos. Nota-se que todos os sistemas apresentaram resultados melhores que o óleo puro. O comportamento não Newtoniano das amostras foi confirmado por meio das análises das curvas da tensão de cisalhamento em função da taxa de cisalhamento na temperatura de $25^{\circ} \mathrm{C}$. As curvas relativas aos sistemas estudados são mostradas na Figura 4. 
Figura 4. Curvas da tensão de cisalhamento em função da taxa de cisalhamento em temperatura de $25^{\circ} \mathrm{C}$ para os óleos estudados

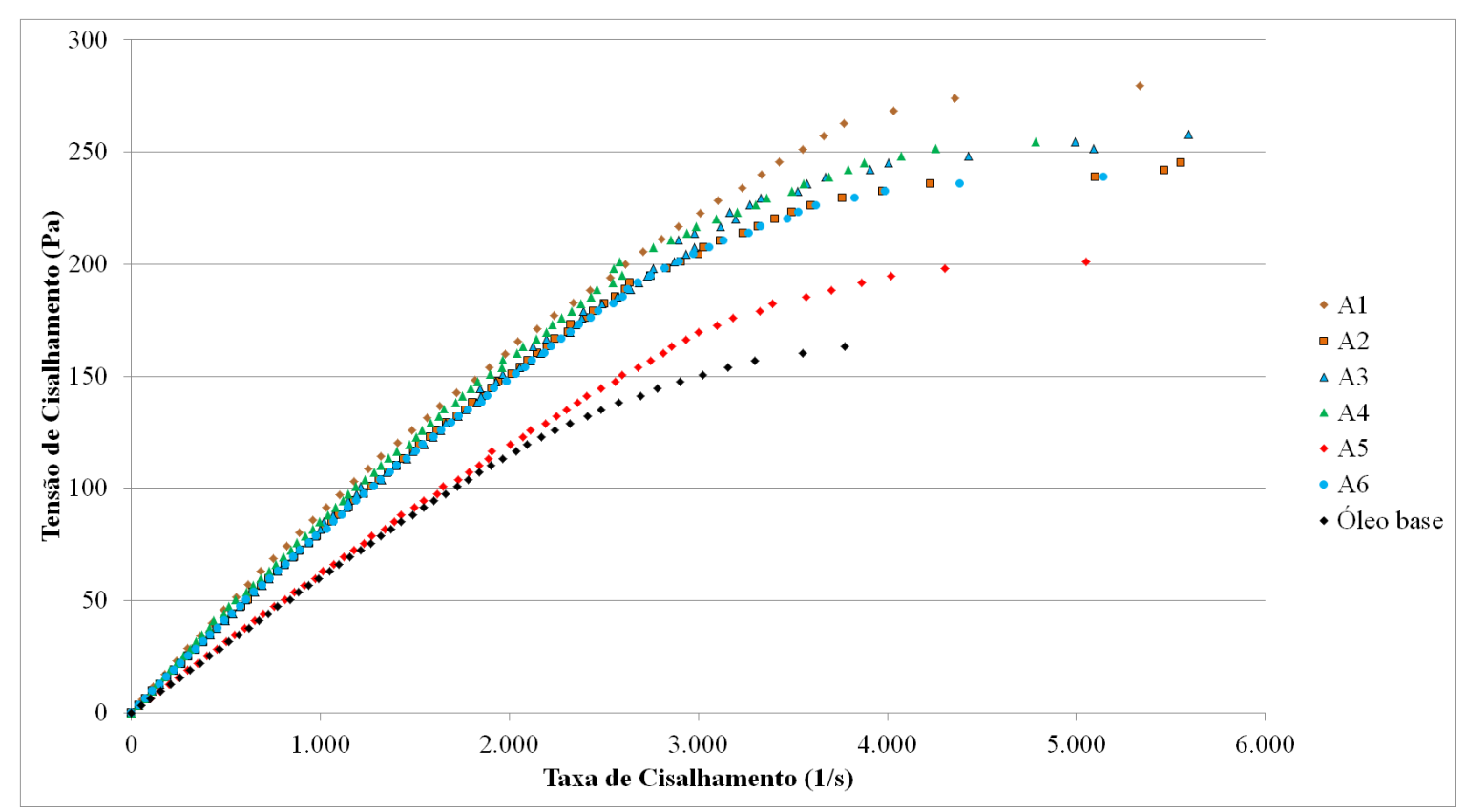

Por meio da análise das curvas (Figura 4) percebe-se a não linearidade entre a tensão de cisalhamento e a taxa de deformação.

Na Tabela 6 são apresentados os resultados para os parâmetros do modelo de potência obtidos para as amostras analisadas.

Tabela 6. Parâmetros do Modelo de Potência para as amostras analisadas

\begin{tabular}{c|c|c|c}
\hline \multicolumn{2}{c}{ Modelo de Potência: $\mathrm{y}=\mathrm{a} . \mathrm{x}$} \\
\hline Parâmetros & A & B & $\mathrm{R}^{2}$ \\
\hline Óleo SN-150 & 0,2375 & 0,8062 & 0,9999 \\
\hline A1 & 0,5083 & 0,7550 & 0,9999 \\
\hline A2 & 0,7023 & 0,6999 & 0,9999 \\
\hline A3 & 0,5554 & 0,7338 & 0,9999 \\
\hline A4 & 0,4537 & 0,7653 & 0,9999 \\
\hline A5 & 0,2741 & 0,7955 & 0,9999 \\
\hline A6 & 0,4559 & 0,7570 & 0,9999 \\
\hline
\end{tabular}


Os resultados mostrados na Tabela 6 ratificam o comportamento não Newtoniano das amostras aditivadas. O modelo que mais se adequou aos dados foi o modelo de Ostwald de Waale ou Modelo de Potência (Lei de Potência), com $b<1$.

\section{CONCLUSÃO}

Neste trabalho foram estudadas seis composições de melhoradores do índice de viscosidade sintetizados a partir de metacrilatos (dodecilmetacrilato e octadecilmetacrilato) e anidrido maleico, utilizando o Luperox ${ }^{\circledR}$ TBEC como iniciador.

A formação dos terpolímeros foi comprovada por meio da técnica de FTIR. Os óleos aditivados apresentaram estabilidade térmica adequada para utilização em motores automotivos com temperaturas iniciais de degradação a partir de $238^{\circ} \mathrm{C}$, valor bem superior ao da temperatura máxima de trabalho desse tipo de equipamento.

Verificou-se que a concentração relativa dos monômeros influencia a estabilidade térmica dos compostos, sendo este efeito mais dependente da concentração de dodecilmetacrilato.

Houve um aumento nas viscosidades cinemáticas dos óleos aditivados a 40 e $100^{\circ} \mathrm{C}$, assim como nos IVs em relação ao óleo puro, conforme esperado para um MIV. As amostras apresentaram valores elevados para os IVs, mesmo após os testes de cisalhamento. Esses resultados são animadores, visto que valores reduzidos de perda por cisalhamento prolongam a vida útil dos óleos aditivados, evitando a perda permanente de viscosidade.

A partir dos ensaios reométricos foi possível verificar que o modelo que melhor descreve o comportamento do fluido é o modelo de potência.

\section{REFERÊNCIAS}

[1] GOSH, Pranab; DAS, Moumita. Synthesis, Characterization, and Performance Evaluation of Some Multifunctional Lub Oil Additives; Journal of Chemical \& Engineering data; University of North Bengal, India, pages 510-516, 2013.

[2] BARROS, BARROS, Guilherme. Mercado e Economia. Disponível em http://guilhermebarros.ig.com.br/2010/09/27/mercado-brasileiro-de-lubrificantes-devecrescer-ate-3-nos-proximos-10-anos-diz-pesquisa/. Acesso em: 14/01/2016.

[3] KRAGULJAC, Kornelije; SOLJIC, Ivana; VIDOVIC, Elvira; JUKIC, Ante. Miscibility and interactions of rheology improvers based on functional polymethacrylate in toluene solutions. Chemical Engineering Transactions, Faculty of Chemical Engineering and Technology, University of Zagreb, Croatia, vol. 17, 2009.

[4] MORTIER, Roy; FOX, Malcolm; ORSZULIK, Stefan; Chemistry and Thecnology of Lubricants. Springer, 2010.

[5] VERDIER, Sylvain; COUTINHO, João; SILVA, Artur; ALKILDE, Ole; HANSEN, Jens; A critical approach to viscosity index; Science Direct, vol. 88, páginas 2199-2206, 2009.

[6] OLIVEIRA, Ana; Terpolímeros como melhoradores de índice de viscosidade de óleos lubrificantes automotivos: Síntese e estudo reológico e termodinâmico; Tese de Doutorado, Universidade Federal de Minas Gerais, Belo Horizonte, 2014. 
[7] GOSH, Pranab; DAS, Moumita; Study of the influence of some polymeric additives as viscosity index improvers and pour point depressants - Synthesis and characterization; Journal of Petroleum Science and Engineering, University of North Bengal, India, 2014.

[8] ALMEIDA, Ana; Caracterização de aditivos poliméricos melhoradores do índice de viscosidade e estudo reológico de lubrificantes automotivos; Dissertação de mestrado, Universidade Federal de Minas Gerais, Belo Horizonte, 2012.

[9] LUCAS, Elisabete; SOARES, Bluma; MONTEIRO, Elisabeth; Caracterização de Polímeros: Determinação de peso molecular e análise térmica; Universidade Federal do Rio de Janeiro, Rio de Janeiro, Editora E-papers, 2001.

[10] COVITCH, Michael ; TRICKETT, Kieran; How polymers behave as viscosity index improvers in lubricating oils; Advances in Chemical Engineering and Science, pages 134$151,2015$.

[11] MORGAN, Shawn; ZHIBIN, Ye; SUBRAMANIAN, Ramesh; ZHU, Shiping; HigherMolecular-Weight Hyperbranched Polyethylenes Containing Crosslinking Structures as Lubricant Viscosity-Index Improvers; Polymer Engineering and Science, McMaster University, Canada, pages 911- 918; 2010.

\section{AGRADECIMENTOS}

Os autores agradecem à Petrobras, à FAPEMIG, à CAPES e ao CNPq. 